\title{
The synthesis problem of the optimum control for nonlinear stochastic structures in the multistructural systems and methods of its solution
}

\author{
Sergey V. Sokolov \\ Rostov State University of means of communication
}

Russia

\section{The problem statement of the optimum structure selection in nonlinear dynamic systems of random structure}

The most interesting and urgent, but unsolved till now, problem in the theory of the dynamic systems of random structure is the synthesis problem for the optimum control of the structure selection in the sense of some known criterion on the basis of the information obtained from the meter. The classical results known in this direction [1] allow to solve the problem of the optimum control only by the system itself (or its specific structure), but not by the selection of the structure.

In this connection the solution of the synthesis problem for the optimum selection control of the structure for the nonlinear stochastic system by the observations of the state vector under the most general assumptions on the character of the criterion applied for the selection optimality is of theoretical and practical interest.

To solve the given problem we formulate it as follows.

For the nonlinear dynamic system of random structure, generally [1] described in the l-th state by the vector equation of form

$$
\dot{\xi}=f^{(l)}(\xi, t)+f_{0}^{(l)}(\xi, t) n_{t}^{(l)}, \quad \xi\left(t_{0}\right)=\xi_{0}
$$

where $l=\overline{1, S}$ is the state number (number of the structure);

$f^{(l)}(\xi, t), f_{0}^{(l)}(\xi, t)$ are nonlinear vector and matrix functions of the appropriate dimension $n^{(l)} \leq N$ and $m^{(l)} \times n^{(l)}, \quad N=\max \left(n^{(1)}, \ldots, n^{(S)}\right)$;

$\xi(t)$ is the state vector of dimension $N$ in any structure,

$n_{t}^{(l)}$ is the Gaussian white normalized vector - noise of dimension $m^{(l)}$; the observer of the state vector of which is described, in its turn, by the equation 


$$
Z=H^{(l)}(\xi, t)+W_{t}^{(l)}
$$

where $Z$ is the $M$ - dimensional vector of the output signals of the meter; $H^{(l)}(\xi, t)$ is the vector - function of the observation of the $l$-th structure of dimension $M$; is the Gaussian white vector - noise with the zero average and matrix of intensities $D_{W}^{(l)}(t)$, we should find such a law of transition $v(\xi, t, Z, l=\overline{1, s})$ from one structure into another, which would provide on the given interval of time $T=\left[t_{0}, t_{K}\right]$ the optimum of some probabilistic functional $J_{0}$ generally nonlinearly dependent on the a posteriori density of distribution $\rho\left(\xi, t / Z(\tau), \tau \in\left[t_{0}, t\right]\right)=\rho(\xi, Z, t)$ of the state vector :

$$
J_{0}=\int_{T} \int_{\xi_{*}} \Phi[\rho(\xi, Z, t)] d \xi d t
$$

where $\xi_{*}$ is the domain of defining argument $\xi$ in which the optimum is searched;

$\Phi$ is the given nonlinear analytical function.

Thus the different versions of the form of function $\Phi$ allow to cover a wide class of the optimality conditions by accuracy of:

- the probability maximum (minimum) of vector $\xi$ in the area $\xi_{*}: \Phi(\rho)= \pm \rho$;

- the deviation minimum of the required probability density $\rho$ from the given one g: $\Phi(\rho)=(\rho-g)^{2}, \Phi(\rho)=|\rho-g|, \Phi(\rho)=-\rho \ln \left(\frac{g}{\rho}\right)$ (the Kulback criterion) etc.;

- the information maximum on the state vector $\xi$ : $\Phi(\rho)=\rho\left[\frac{\partial \ln \rho}{\partial \xi}\right]\left[\frac{\partial \ln \rho}{\partial \xi}\right]^{T}$ (the Fisher criterion ) etc.

The similar formulation of the problem covers the selection problem for the optimum structure and in the nonobservable stochastic systems of random structure as well - in this case in the expression for $J_{0}$ by $\rho$ we understood the prior density of vector $\xi$. Thus the form of function $\Phi$ should be selected taking into account, naturally, the physical features of the problem solved.

The analysis of the physical contents of the structure selection control providing the optimum of functional $J_{0}$ shows that as the vector determining subsequently the control of the structural transitions, it is most expedient to use the vector of intensities of the state change $[1,2]$

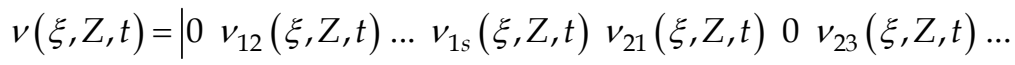

$$
\begin{aligned}
& \left.v_{2 s}(\xi, Z, t) v_{31}(\xi, Z, t) v_{32}(\xi, Z, t) 0 v_{34}(\xi, Z, t) \ldots v_{s(s-1)}(\xi, Z, t) 0\right|^{T} \text {, }
\end{aligned}
$$

where $v_{l r}(\xi, Z, t)$ is the intensity of transitions from state $l$ into state $r$, requiring while its forming, for example, in order to prevent the frequent state change, the minimum of its quadratic form on the given interval of time $T$ for $\xi \in \xi *$, i.e. 


$$
\min \int_{T} \int_{\xi_{*}} v^{T}(\xi, Z, t) v(\xi, Z, t) d \xi d t
$$

As far as vector $v$ contains the zero components, then in essence, from here on the search not of vector $v$ itself, but vector $v_{0}$, related to it by the relationship $v=E_{0} v_{0}$, is carried out, where $v_{0}$ is the vector formed from vector $v$ by eliminating the zero components;

$E_{0}$ is the matrix formed from the unit one by adding the zero rows to form the appropriate zero elements in vector $v$.

And finally the minimized criterion $J$ takes the form

$$
J=\int_{T} \int_{\xi_{*}}\left(\Phi[\rho(\xi, Z, t)]+v_{0}^{T}(\xi, Z, t) v_{0}(\xi, Z, t)\right) d \xi d t
$$

In its turn, for process $\xi$ described by equations (1), the density of its a posteriori distribution $\rho$ (DAPD) can be given as

$$
\rho(\xi, Z, t)=\sum_{l=1}^{s} \omega(\xi, Z, l, t)=\sum_{l=1}^{s} \omega_{Z}^{(l)}(\xi, t),
$$

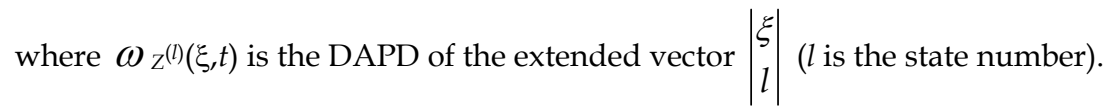

In the case of the continuous process $\xi$, which is most typical for practice, when the restored values of the $l$-th state coincide with the final value of the process of the $r$-th state, functions $\omega_{Z^{(l)}}(\xi, t), l=\overline{1, S}$, are described by the following system of the Stratonovich generalized equations [1]:

$$
\begin{gathered}
\frac{\partial \omega_{Z}^{(l)}(\xi, t)}{\partial t}=L\left[\omega_{Z}^{(l)}(\xi, t)\right]+Q\left[\omega_{Z}^{(l)}(\xi, t)\right]-\sum_{r=1}^{s} v_{l r}(\xi, t) \omega_{Z}^{(l)}(\xi, t)+\sum_{r=1}^{s} v_{r l}(\xi, t) \omega_{Z}^{(r)}(\xi, t), \\
l=\overline{1, s}, \\
Q\left[\omega_{Z}^{(l)}(\xi, t)\right]=-\frac{1}{2} \omega_{Z}^{(l)}(\xi, t)\left[\gamma^{(l)}(\xi, Z, t)-\sum_{k=1}^{s} \int_{-\infty}^{\infty} \gamma^{(l)}(\xi, Z, t) \omega_{Z}^{(k)}(\xi, t) d \xi\right], \\
\gamma^{(l)}(\xi, Z, t)=\sum_{p, q=1}^{M} \frac{\hat{D}_{p q}^{(l)}(t)}{\left|D_{W}^{(l)}(t)\right|}\left[Z_{p}-H_{p}^{(l)}(\xi, t)\right]\left[Z_{q}-H_{q}^{(l)}(\xi, t)\right],
\end{gathered}
$$

$\hat{D}_{p q}^{(l)}(t)$ is the algebraic addition of the pq-th element in the determinant $\left|D_{W}^{(l)}(t)\right|$ of matrix $D_{W}^{(l)}(t)$;

$p, q$ are indexes of the respective components of vectors; 
$L \quad$ is the Fokker -Planck (FP) operator;

or entering vector $v_{0}(\xi, Z, t)$ and vector $\omega_{Z}(\xi, t)=\left|\omega_{Z}^{(1)}(\xi, t) \ldots \omega_{Z}^{(s)}(\xi, t)\right|^{T}$, we have in the general form:

$$
\begin{gathered}
\frac{\partial \omega_{Z}(\xi, t)}{\partial t}=U\left[\omega_{Z}(\xi, t)\right]-\left[\Omega\left[\omega_{Z}(\xi, t)\right]\left(E_{s} \otimes I_{s}\right)-\omega_{Z}^{T}(\xi, t) \otimes E_{s}\right] E_{0} v_{0}(\xi, Z, t), \\
U\left[\omega_{Z}\right]=L\left[\omega_{Z}\right]+Q\left[\omega_{Z}\right],
\end{gathered}
$$

where $E_{S}$ is the unit matrix of dimension $S$;

$I_{S}$ is the unit row of dimension $S$;

$\otimes \quad$ is the symbol of the Kronecker product;

$$
\Omega\left(\omega_{Z}\right)=\left|\begin{array}{ccc}
\omega_{Z}^{(1)} & & 0 \\
& \omega_{Z}^{(2)} & \\
& \cdots & \\
0 & & \omega_{Z}^{(s)}
\end{array}\right| .
$$

For the nonobservable dynamic systems the FP generalized equations are derived from (4) at $Q=0$.

Taking into account that introducing vector $\omega_{Z}$ for density $\rho$ the expression has the form

$$
\rho(\xi, Z, t)=I_{s} \omega_{Z}(\xi, t)
$$

functional (3) is given as

$$
J=\int_{T} \int_{\xi_{*}}\left[\Phi\left[I_{S} \omega_{Z}(\xi, t)\right]+v_{0}^{T}(\xi, Z, t) v_{0}(\xi, Z, t)\right] d \xi d t=\int_{T} W_{*}(t) d t,
$$

and for the simplification of the subsequent solution the vector equation (4) is rewritten as follows:

$$
\frac{\partial \omega_{Z}}{\partial t}=U\left(\omega_{Z}\right)-\left[\Omega\left(\omega_{Z}\right)\left(E_{S} \otimes I_{S}\right)-\omega_{Z}^{T} \otimes E_{S}\right] E_{0} v_{0}=U\left(\omega_{Z}\right)-F\left(\omega_{Z}\right) v_{0}
$$

Then the problem stated finally can be formulated as the problem of search of vector $V_{0}$, that provides the synthesis of such a vector $\omega_{Z}$ described by equation (6), which would deliver the minimum to functional (5). The synthesis of the optimum vector $\omega_{z}$ allows immediately 
to solve the problem of the selection of the optimum structure by defining the maximal components of the vector of the state probabilities $P(t)=\int_{-\infty}^{\infty} \omega_{Z}(\xi, t) d \xi[1]$.

\section{The general solution of the synthesis problem for the stochastic structure control}

For the further solution of the problem we use the method of the dynamic programming, according to which by search of the optimum control in the class of the limited piecewisecontinuous functions with values from the open area $v *$ the problem is reduced to the solution of the functional equation [3]

$$
\min _{v \in V_{*}}\left\{\frac{d V}{d t}+W_{*}\right\}=0
$$

under the final condition $V\left(t_{K}\right)=0$ with respect to optimum functional $V$, parametrically dependent on time $t \in T$ and determined on a set of vector - functions $\omega_{\mathrm{z}}$, satisfying equation (6).

For the linear systems functional $V$ is found as the integrated quadratic form [3]

$$
V=\int_{\xi_{*}} \omega_{Z}^{T}(\xi, t) \mathrm{v}(\xi, t) \omega_{z}(\xi, t) d \xi
$$

$\mathrm{v}$ is a $S \times S$ matrix, whence we have:

$$
\frac{d V}{d t}+W_{*}=\int_{\xi_{*}}\left\{\omega_{Z}^{T} \frac{\partial \mathrm{v}}{\partial t} \omega_{Z}+\omega_{Z}^{T}\left(\mathrm{v}^{T}+\mathrm{v}\right) \frac{\partial \omega_{Z}}{\partial t}+\Phi\left(I_{S} \omega_{Z}\right)+v_{0}^{T} v_{0}\right\} d \xi,
$$

and taking into account equation (6) for $\omega_{Z}$ we obtain the initial expression for the subsequent definition of the optimum one $v_{0}{ }^{*}$

$$
\frac{d V}{d t}+W_{*}=\int_{\xi_{*}}\left\{\omega_{Z}^{T} \frac{\partial \mathrm{v}}{\partial t} \omega_{Z}+\omega_{Z}^{T}\left(\mathrm{v}^{T}+\mathrm{v}\right)\left(U\left(\omega_{Z}\right)-F\left(\omega_{\mathrm{Z}}\right) v_{0}\right)+\Phi\left(I_{S} \omega_{Z}\right)+v_{0}^{T} v_{0}\right\} d \xi
$$

The analysis of the given expression shows that the definition of vector $v_{0}{ }^{*}$ from the solution of the functional equation (7) is reduced to the classical problem of search of vector - function realizing the minimum of the certain integral (8). Thus the required vector - function $v_{0}^{*}(\xi, Z, t)$ should satisfy the following system of the Euler equations:

whence

$$
-F^{T}\left(\omega_{\mathrm{Z}}\right)\left(\mathrm{v}^{T}+\mathrm{v}\right) \omega_{\mathrm{Z}}+2 v_{0}^{*}=0
$$




$$
v_{0}^{*}=\frac{1}{2} F^{T}\left(\omega_{\mathrm{Z}}\right)\left(\mathrm{v}^{T}+\mathrm{v}\right) \omega_{\mathrm{Z}} .
$$

The substitution of the found optimum law for the change of state $v_{0}{ }^{*}$ into (6) allows to write down the equations for the optimum (in the sense of (5)) vector $\omega_{Z}$

$$
\frac{\partial \omega_{Z}}{\partial t}=U\left(\omega_{Z}\right)-\frac{1}{2} F\left(\omega_{Z}\right) F^{T}\left(\omega_{Z}\right)\left(v^{T}+v\right) \omega_{Z}
$$

the integration of which, in its turn, completes the solution of the selection problem of the optimum structure by defining the maximal component of the vector of the state probabilities

$$
P(t)=\int_{-\infty}^{\infty} \omega_{z}(\xi, t) d \xi
$$

The equations required for defining the matrix function $\mathrm{v}(\xi, t)$, included in (9), follow from the constrain

$$
\frac{d V}{d t}+W_{*}=\left.0\right|_{v_{0}=v_{0}^{*}}
$$

after substituting the found vector $v_{0}{ }^{*}$ into (8)

$$
\frac{\partial \mathrm{v}}{\partial t}=-\frac{1}{s}\left(\mathrm{v}^{T}+\mathrm{v}\right) U\left(\omega_{\mathrm{Z}}\right) \omega_{0}^{T}-\frac{1}{s^{2}} \omega_{0} \omega_{0}^{T} \Phi\left(I_{S} \omega_{\mathrm{Z}}\right)+\frac{1}{4}\left(\mathrm{v}^{T}+\mathrm{v}\right) F\left(\omega_{\mathrm{Z}}\right) F^{T}\left(\omega_{\mathrm{Z}}\right)\left(\mathrm{v}^{T}+\mathrm{v}\right),
$$

where $\omega_{0}=\left|\frac{1}{\omega_{Z}^{(1)}} \ldots \frac{1}{\omega_{Z}^{(S)}}\right|^{T}$ is the auxiliary vector introduced for convenience in transformations and simplification of the record of equation (10).

The joint solution of systems $(9,10)$ under the boundary-value constrains $\omega_{z}\left(\xi, t_{0}\right)=\omega_{z 0}, \mathrm{v}\left(\xi, t_{k}\right)=0$ exhausts, in essence, the theoretical solution of the problem stated, including the prior case as well - for the nonobservable dynamic systems of random structure.

It should be noted that in case of forming $v_{0}{ }^{*}$ in the assumption of its independence on $\xi$, the integrated dependence $v_{0}{ }^{*}$ on $\omega_{Z}$ follows from the constrain of minimization of the functional equation (8)

$$
v_{0}^{*}=(2 \Delta)^{-1} \int_{\xi_{*}} F^{T}\left(\omega_{z}\right)\left(\mathrm{v}^{T}+\mathrm{v}\right) \omega_{z} d \xi, \Delta=\int_{\xi_{*}} d \xi,
$$

which after substitution into (6) and (8) results in forming a system already of the integrodifferential (as distinct from $(9,10)$ ) equations with partial derivatives, the solution search of which appears to be much more difficult than in the first case. 
In spite of the fact that the found theoretical solution of the problem stated defines the basic feasibility of the optimum selection of the process structure $\xi$, the practical solution of the boundary-value problem directly for the conjugated system of equations with the partial derivatives $(9,10)$ represents a problem now.

Then in this connection as one of the solution methods of this problem (as the most universal one) we consider the method using the expansion of functions $\omega_{\mathrm{z}}, \mathrm{v}$ into series by some system of the orthonormal functions $\phi=\left|\phi_{1} \ldots \phi_{N}\right|^{T}$ of vector argument:

$\mathrm{v}(\xi, t)=B(t) \phi(\xi), \omega_{z}(\xi, t)=A(t) \phi(\xi) ; A(t), B(t)$ are ordinary and block matrixes of factors of expansion determined in the course of solving. In this case the problem is reduced to the point-to-point boundary-value problem of integration of the matrix system already of the ordinary differential equations:

$$
\begin{gathered}
\frac{\partial A}{\partial t}=\int_{\xi}\left\{U(A \phi) \phi^{T}-\frac{1}{2} F(A \phi) F^{T}(A \phi)\left(\phi^{T} B^{T}+B \phi\right)(A \phi) \phi^{T}\right\} d \xi, \\
\frac{\partial B}{\partial t}=-\frac{1}{S} \int_{\xi}\left\{\left(\phi^{T} B^{T}+B \phi\right) U(A \phi) \omega_{0}^{T}(A \phi)-\frac{1}{S^{2}} \omega_{0}(A \phi) \omega_{0}^{T}(A \phi) \Phi\left(I_{S} A \phi\right)+\right. \\
\left.+\frac{1}{4}\left(\phi^{T} B^{T}+B \phi\right) F(A \phi) F^{T}(A \phi)\left(\phi^{T} B^{T}+B \phi\right)\right\} \phi^{T} d \xi
\end{gathered}
$$

the solution of which appears to be already much easier and can be carried out by various traditional ways : the ranging short method, the method of the invariant plunge etc. The feature of the practical realization of the solution in this case is the absence of the rigid requirements to its accuracy, as in case of the structure selection the number of the maximal components of vector $P(t)=A(t) \int_{\xi} \phi(\xi) d \xi$, rather than its value is only defined. For the illustration of the real feasibility of applying the similar approach we consider the example. For the nonlinear stochastic process of random structure described by the equation

$$
\dot{\xi}=f^{(l)}(\xi, t)+n_{t}
$$

where $l=1,2 ; f^{(1)}(\xi, t)=-\xi^{2}, f^{(2)}(\xi, t)=-\xi+0,01 \xi^{3}$, $n_{t}$ is the Gaussian normalized white noise, the equation of the observer has the form

$$
Z=H^{(l)}(\xi, t)+W_{t}
$$

where $l=1,2 ; H^{(1)}(\xi, t)=0,5 \xi^{2}, H^{(2)}(\xi, t)=1,2 \xi^{2}-0,1 \xi^{3}$;

$W_{t}$ is the Gaussian normalized white noise.

It is required to carry out the structure selection of process $\xi$ providing the maximum of probability of its occurrence in the given limits $\xi_{*}=\left[\xi_{\min }=-0,5 ; \xi_{\max }=0,7\right]$ in time interval $T=[0 ; 300]$ s., i.e. the minimizing criterion 


$$
J=\int_{T} \int_{\xi_{\star}}\left\{-\rho(\xi, t)+v_{0}^{T}(\xi, t) v_{0}(\xi, t)\right\} d \xi d t
$$

where

$$
\begin{gathered}
v_{0}=\left|\begin{array}{l}
v_{12} \\
v_{21}
\end{array}\right| ; \quad \rho(\xi, t)=\omega_{z}^{(1)}(\xi, t)+\omega_{z}^{(2)}(\xi, t) ; \\
\frac{\partial \omega_{z}^{(1)}}{\partial t}=\frac{\partial}{\partial \xi}\left(\xi^{2} \omega_{z}^{(1)}\right)+\frac{1}{2} \frac{\partial^{2} \omega_{z}^{(1)}}{\partial \xi^{2}}- \\
-\frac{1}{2} \omega_{z}^{(1)}(\xi, t)\left[\left(Z-0,5 \xi^{2}\right)^{2}-\sum_{k=1}^{2} \int_{-\infty}^{\infty}\left(Z-0,5 x^{2}\right)^{2} \omega_{z}^{(k)}(x, t) d x\right]-v_{12} \omega_{z}^{(1)}+v_{21} \omega_{z}^{(2)} ; \\
\frac{\partial \omega_{z}^{(2)}}{\partial t}=\frac{\partial}{\partial \xi}\left[\left(\xi-0,01 \xi^{3}\right) \omega_{z}^{(2)}\right]+\frac{1}{2} \frac{\partial^{2} \omega_{z}^{(2)}}{\partial \xi^{2}}-\frac{1}{2} \omega_{z}^{(2)}(\xi, t)\left[\left(Z-1,2 \xi^{2}+0,1 \xi^{3}\right)^{2}-\right. \\
\left.-\sum_{k=1}^{2} \int_{-\infty}^{\infty}\left(Z-1,2 x^{2}+0,1 x^{3}\right)^{2} \omega_{z}^{(k)}(x, t) d x\right]-v_{21} \omega_{z}^{(2)}+v_{12} \omega_{z}^{(1)},
\end{gathered}
$$

or in the vector form

$$
\begin{gathered}
\frac{\partial \omega_{z}}{\partial t}=U\left(\omega_{z}\right)-F\left(\omega_{z}\right) v_{0}, \quad \omega_{z}=\left|\omega_{z}^{(1)} \omega_{z}^{(2)}\right|^{T}, \\
F\left(\omega_{z}\right)=\left|\begin{array}{cc}
\omega_{z}^{(1)} & -\omega_{z}^{(2)} \\
-\omega_{z}^{(1)} & \omega_{z}^{(2)}
\end{array}\right| .
\end{gathered}
$$

In this case equations $(9,10)$ for the optimum vector $\omega_{Z}$ and conjugated matrix function $V$ have the form:

$$
\begin{aligned}
& \frac{\partial \omega_{z}}{\partial t}=U\left(\omega_{z}\right)-\frac{1}{2}\left|\begin{array}{cc}
\Omega_{0} & -\Omega_{0} \\
-\Omega_{0} & \Omega_{0}
\end{array}\right|\left(V^{T}+V\right) \omega_{z}, \\
& \frac{\partial V}{\partial t}=-\frac{1}{2}\left(V^{T}+V\right) U\left(\omega_{z}\right)\left|\frac{1}{\omega_{z}^{(1)}} \frac{1}{\omega_{z}^{(2)}}\right|+ \\
& +\frac{1}{4}\left|\begin{array}{cc}
\frac{\omega_{z}^{(1)}+\omega_{z}^{(2)}}{\omega_{z}^{(1) 2}} & \frac{\omega_{z}^{(1)}+\omega_{z}^{(2)}}{\omega_{z}^{(1)} \omega_{z}^{(2)}} \\
\frac{\omega_{z}^{(1)}+\omega_{z}^{(2)}}{\omega_{z}^{(1)} \omega_{z}^{(2)}} & \frac{\omega_{z}^{(1)}+\omega_{z}^{(2)}}{\omega_{z}^{(2) 2}}
\end{array}\right|+\frac{1}{4}\left(V^{T}+V\right)\left|\begin{array}{cc}
\Omega_{0} & -\Omega_{0} \\
-\Omega_{0} & \Omega_{0}
\end{array}\right|\left(V^{T}+V\right), \\
& \Omega_{0}=\omega_{z}^{(1) 2}+\omega_{z}^{(2) 2} \text {. }
\end{aligned}
$$


The solution of the given problem was carried out on the basis of the approximation of functions $\omega_{\mathrm{Z}}, V$ by the Fourier series in interval $[-5 ; 5]$ within the accuracy of 4 terms of expansion and integration of the obtained system of equations for factors of expansion by means of the approximated method of the invariant plunge on time interval $[0 ; 300] \mathrm{s}$. When integration and formation of the approximated values of functions $\omega_{Z^{(1)}}, \omega_{Z^{(2)}}$ had been taken place, the numbers of the structures selected by the character of the maximal state probability at the current time, appeared to be distributed in time as follows:

- in interval $[0 ; 48] \mathrm{s}$ - the second structure;

- in interval $[48 ; 97] \mathrm{s}$ - the first structure;

- in interval [97; 300] s - the second structure.

Concurrently the integration of the system of the DAPD equations $\omega_{Z^{(1)}}$,

$\omega_{Z^{(2)}}$ was carried out for the traditional case of the uncontrolled change of states with the unit intensity [1] and it was established that in the latter case the value of the minimized criterion $J$ had appeared to be by a factor of 1,35 higher than that of in the optimum control of the structure selection.

Thus it allows to make a conclusion not only on the theoretical solution of the general problem for the optimum structure control of the stochastic dynamic systems, but on the feasibility of the effective practical application of the method developed as well. It is obvious that for the dynamic systems of the great dimension again there arises the traditional problem of the numerical realization of the approach suggested. As far as one of the ways of simplifying the control algorithms lays in the direction of the preliminary solution approximation of the vector equation of the distribution density, then for the multistructural system we apply the approach suggested in [1], allowing to write down the differential equations of the parameters of density, approximating the initial one. Thus it should be taken into account the character of the approximation, which arises only in the multistructural system, - only the normalized densities of the state vectors of each structure (ignoring the probabilities of occurring the structures themselves) are approximated.

In this case the function of the distribution density $\xi$ of the state vector can be given as [1]

$$
\rho(\xi, t)=\sum_{l=1}^{S} P_{l} \rho_{l}(\xi, t),
$$

where $P_{l}$ is the probability of the $l$-th structure;

$\rho_{l}(\xi, t)$ is the distribution density $\xi$ in the $l$-th structure.

Then the scheme of the synthesis of the structure control, following from the similar approach, we consider in detail. Thus we investigate the prior case (for the nonobservable structures), as a more adequate one to the practical applications (due to the character of forming the terminal control requiring the whole set of measurements on the given time interval of optimization, that for a number of practical problems is unacceptable). But it should be noted that the synthesis of the a posteriori control on the basis of the approach described below does not practically differ from the prior one - only by the additional component caused by the observation availability in the right-hand part of the equations of parameters (12) [1] given below (not affecting the procedure of forming the control required). So, we investigate the synthesis method for the structure control using the approximating representation of the densities of the state vectors of the dynamic structures. 


\section{Suboptimum selection control of nonlinear dynamic structures}

In solving the practical problems of the analysis and synthesis of systems with random structure to describe densities $\rho_{l}$ one uses, as a rule, their Gaussian approximation $\tilde{\rho}_{l}$ which results in specifying the parameters determining $\tilde{\rho}_{l}$ - the vector of the mathematical expectation $\hat{\xi}^{(l)}$ and the covariance matrix $R^{(l)}$, in the form of the known system of the ordinary differential equations (providing the required trade-off of accuracy against the volume of the computing expenses [4]).

In the case investigated below for the continuous process $\xi$, when the restored values of the $l$-th state coincide with the final values of the process of the $r$-th state, the system of equations for parameters $\tilde{\rho}$, obtained on the basis of the Gaussian approximation $\rho$, has the following form $[1,4]$ :

$$
\begin{gathered}
\hat{\dot{P}}_{l}=-\sum_{r=1}^{S}\left(\hat{P}_{l} v_{l r}\left(\hat{\xi}^{(l)}, R^{(l)}, t\right)-\hat{P}_{r} v_{r l}\left(\hat{\xi}^{(r)}, R^{(r)}, t\right)\right) \\
\hat{\dot{\xi}}^{(l)}=f^{(l)}\left(\hat{\xi}^{(l)}, t\right)+\sum_{r=1}^{S} \frac{\hat{P}_{r}(t)}{\hat{P}_{l}(t)} v_{r l}\left(\hat{\xi}^{(r)}, R^{(r)}, t\right)\left[\hat{\xi}^{(r)}-\hat{\xi}^{(l)}\right], \\
\dot{R}^{(l)}=R^{(l)} \frac{\partial f^{(l)}}{\partial \hat{\xi}}\left(\hat{\xi}^{(l)}, t\right)+\frac{\partial f^{(l) T}}{\partial \hat{\xi}}\left(\hat{\xi}^{(l)}, t\right) R^{(l)}+f_{0}^{(l)}\left(\hat{\xi}^{(l)}, t\right) f_{0}^{(l)^{T}}\left(\hat{\xi}^{(l)}, t\right)+ \\
+\sum_{r=1}^{S} \frac{\hat{P}_{r}(t)}{\hat{P}_{l}(t)} v_{r l}\left(\hat{\xi}^{(r)}, R^{(r)}, t\right)\left(R^{(r)}-R^{(l)}+\left(\hat{\xi}^{(r)}-\hat{\xi}^{(l)}\right)\left(\hat{\xi}^{(r)}-\hat{\xi}^{(l)}\right)^{T}\right) \\
l=\overline{1, S},
\end{gathered}
$$

$\hat{P}_{l}$ is the probability of the $l$-th process structure under the Gaussian approximation; $v_{l r}\left(\hat{\xi}^{(l)}, R^{(l)}, t\right)$ is the intensity of transitions from state $l$ into state $r$.

To find the solution required in the general form we present the given system as follows. Introduce vectors

$$
\hat{P}=\left|\hat{P}_{1} \ldots \hat{P}_{S}\right|^{T}, \quad \hat{\xi}=\left|\hat{\xi}^{(1) T} \ldots \hat{\xi}^{(S) T}\right|^{T}
$$

and using the operation of transformation of matrix $A$ with components $a_{i j}(i, j=m, n)$ into vector $A(\mathrm{v})$ :

$$
A^{(v)}=\left|a_{11} a_{21} \ldots a_{m 1} a_{12} a_{22} \ldots a_{m 2} \ldots a_{1 n} a_{2 n} \ldots a_{m n}\right| \mathrm{T},
$$

we previously write down the equations of parameters $\tilde{\rho}$, separating the components into dependent and independent ones on intensities $v_{r l}$ : 


$$
\begin{aligned}
& \hat{\dot{P}}=\left|\begin{array}{c}
\sum_{r=1}^{S}\left(\hat{P}_{r} v_{r l}-\hat{P}_{l} v_{l r}\right) \\
\hdashline \sum_{r=1}^{S}\left(\hat{P}_{r} v_{r S}-\hat{P}_{S} v_{S r}\right)
\end{array}\right|, \quad \hat{\dot{\xi}}=\beta(\hat{\xi}, t)+\left|\begin{array}{l}
\sum_{r=1}^{S} G_{r l} v_{r l} \\
\sum_{r=1}^{S} G_{r S} v_{r S}
\end{array}\right|, \\
& \dot{R}^{(v)}=\Psi\left(\hat{\xi}, R^{(v)}, t\right)+\left|\begin{array}{c}
\sum_{r=1}^{S} Q_{r l} v_{r l} \\
\hdashline \sum_{r=1}^{S} Q_{r S} v_{r S}
\end{array}\right| \\
& R^{(v)}=\left|\begin{array}{l}
R^{(1)(v)} \\
-R^{(\bar{S})(v)}
\end{array}\right|, \quad G_{r l}=G_{r l}(\hat{\xi}, \hat{P})=\frac{\hat{P}_{r}}{\hat{P}_{l}}\left(\hat{\xi}^{(r)}-\hat{\xi}^{(l)}\right) \\
& Q_{r l}=Q_{r l}\left(\hat{\xi}, R^{(v)}, \hat{P}\right)=\left(R^{(r)}-R^{(l)}+\left(\hat{\xi}^{(r)}-\hat{\xi}^{(l)}\right)\left(\hat{\xi}^{(r)}-\hat{\xi}^{(l)}\right)^{T}\right)^{(v)} \frac{\hat{P}_{r}}{\hat{P}_{l}},
\end{aligned}
$$

and then unifying vectors $\hat{\xi}$ and $R(\mathrm{~V})$ into the generalized vector $\hat{X}=\left|\begin{array}{c}\hat{\xi} \\ R^{(\mathrm{V})}\end{array}\right|$ as well.

Then as the vector, determining the structural transition control in the system examined, we use, similarly to the above-stated, the vector of intensities of the state change

$$
\begin{gathered}
v(\hat{P}, X, t)=\left|\begin{array}{lll}
0 & v_{21}(\hat{P}, X, t) \ldots v_{S 1}(\hat{P}, X, t) v_{12}(\hat{P}, X, t) 0 v_{32}(\hat{P}, X, t) \ldots \\
v_{S 2}(\hat{P}, X, t) v_{13}(\hat{P}, X, t) v_{23}(\hat{P}, X, t) 0 v_{43}(\hat{P}, X, t) \ldots v_{(S-1) S}(\hat{P}, X, t) 0
\end{array}\right|^{T},
\end{gathered}
$$

that results in the following form of the minimized criterion $\mathrm{J}$ :

$$
J=\int_{T} \int_{\xi_{\star}} \Phi[\tilde{\rho}(\xi, \hat{P}, X, t)] d \xi d t+\int_{T} v_{0}^{T}(\hat{P}, X, t) v_{0}(\hat{P}, X, t) d t
$$

For the problem solution in view of the designations accepted the system of equations (13) is given as:

$$
\begin{gathered}
\hat{\dot{P}}=\left[\left(E \otimes \hat{P}^{T}\right)-\hat{P}\left(E \otimes I_{S}\right) E_{1}\right] E_{0} v_{0}, \\
\dot{X}=\varphi(X, t)+T(\hat{P}, X) E_{0} v_{0},
\end{gathered}
$$

where $\otimes$ is the symbol of the Kronecker product; 
$I_{S}$ is the unit row of dimension $S$;

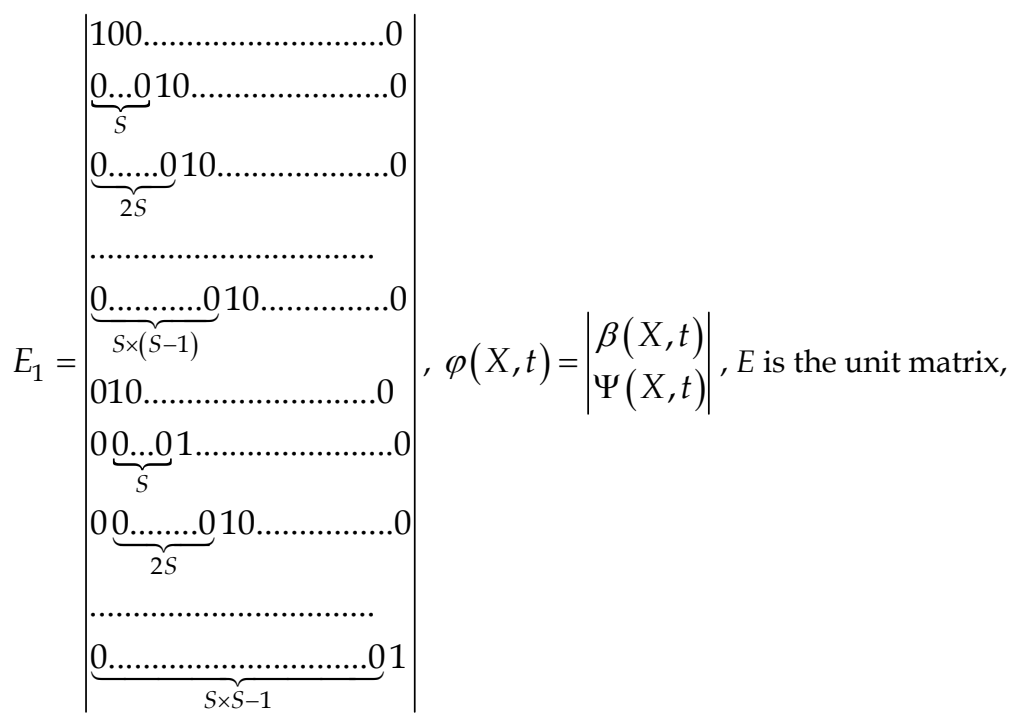

$$
T(\hat{P}, X)=\left|\begin{array}{ccccc}
G_{11} \ldots G_{S 1} & & & \\
& G_{12} \ldots G_{S 2} & 0 & \\
0 & & \ddots & \\
& & & \\
& & G_{1 S} \ldots G_{S S} \\
Q_{11} \ldots Q_{S 1} & & & \\
& Q_{12} \ldots Q_{S 2} & 0 & \\
0 & & \ddots & \\
& & & Q_{1 S} \ldots Q_{S S}
\end{array}\right|,
$$

or finally in the canonical form:

$$
\begin{gathered}
\left|\begin{array}{c}
\hat{\dot{P}} \\
\dot{X}
\end{array}\right|=A(X, t)+B(\hat{P}, X) v_{0}, \quad A(X, t)=\left|\begin{array}{c}
0 \\
\varphi(X, t)
\end{array}\right|, \\
B(\hat{P}, X)=\left|\begin{array}{c}
(E \otimes \hat{P})^{T}-\hat{P}\left(E \otimes I_{S}\right) E_{1} \\
T(\hat{P}, X)
\end{array}\right| E_{0} .
\end{gathered}
$$

Thus the solution of the problem stated is reduced, first, to the definition of vector $v_{0}$ in (16), providing the minimum of functional (14) (in view of the use in the latter as the density 
function its approximation $\tilde{\rho}(\xi, \hat{P}, X, t)$, in this case a Gaussian one), and, secondly, to the selection of the maximal component in vector $\hat{P}$ the number of which will define the number of the required structure of the state vector.

The first stage of the solution - the definition of the optimum vector $v_{0}$ can be carried out by means of the principle of the maximum [5]. In this case the Hamiltonian $H *$ has the form

$$
\begin{aligned}
H_{*}(\hat{P}, X, t)= & \int_{\xi_{*}} \Phi[\tilde{\rho}(\xi, \hat{P}, X, t)] d \xi+v_{0}^{T}(\hat{P}, X, t) v_{0}(\hat{P}, X, t)+ \\
& +\lambda^{T}\left\{A(X, t)+B(\hat{P}, X) v_{0}(\hat{P}, X, t)\right\},
\end{aligned}
$$

$\lambda$ is the vector of the conjugate variables;

whence from the stationarity condition $H_{*}$ the optimum vector $v_{0}{ }^{*}$ is defined directly:

$$
v_{0}^{*}=-\frac{1}{2} B(\hat{P}, X)^{T} \lambda
$$

The substitution of vector $v_{0}^{*}$ into (16) and conjugate system of equations results in the need for solving the point-to-point boundary-value problem for the following system of equations:

$$
\begin{aligned}
& \left|\begin{array}{c}
\hat{\dot{P}} \\
\dot{X}
\end{array}\right|=A(X, t)-\frac{1}{2} B(\hat{P}, X) B^{T}(\hat{P}, X) \lambda, \\
& \dot{\lambda}=-\int_{\xi_{*}}\left|\frac{\partial \Phi(\xi, \hat{P}, X, t)}{\partial \hat{P}} \frac{\partial \Phi(\xi, \hat{P}, X, t)}{\partial X}\right|^{T} d \xi- \\
& -\left(\left|0 \frac{\partial A(X, t)}{\partial X}\right|^{T}-\frac{1}{2} \lambda^{T} B(\hat{P}, X)\left|\frac{\partial B(\hat{P}, X)}{\partial \hat{P}} \frac{\partial B(\hat{P}, X)}{\partial X}\right|^{T}\right) \lambda, \\
& P\left(t_{0}\right)=\hat{P}_{0}, \quad X\left(t_{0}\right)=X_{0}, \quad \lambda\left(t_{k}\right)=0,
\end{aligned}
$$

the integration of which exhausts the theoretical solution of the problem stated- the subsequent selection of the maximal component of vector $\hat{P}$, determining the current number of the required structure of the state vector, does not represent any problem.

Moreover, as above, in selecting the structure the exact value of the maximal component of vector $\hat{P}$ is not essentially required, only its number is of importance. In practical solving of problem (17) this allows to use the approximated methods focused on the required trade-off of accuracy against the volume of the calculations, for example, already approved method of 
the approximated invariant plunge, transforming system (17) into the system of the ordinary differential equations solved in the real time [6]:

$$
\begin{gathered}
\left|\begin{array}{c}
\hat{P}_{*} \\
\dot{X}_{*}
\end{array}\right|=A\left(X_{*}, t\right)-D(t) \int_{\xi *}\left|\frac{\partial \Phi\left(\xi, \hat{P}_{*}, X_{*}, t\right)}{\partial \hat{P}_{*}} \frac{\partial \Phi\left(\xi, \hat{P}_{*}, X_{*}, t\right)}{\partial X_{*}}\right|^{T} d \xi, \\
\dot{D}=2\left|0 \frac{\partial A\left(X_{*}, t\right)}{\partial X_{*}}\right| D+D\left|0 \frac{\partial A\left(X_{*}, t\right)}{\partial X_{*}}\right|^{T}+ \\
+\frac{1}{2} B\left(\hat{P}_{*}, X_{*}\right) B^{T}\left(\hat{P}_{*}, X_{*}\right)-2 D \int \mid \frac{\partial^{2} \Phi\left(\xi, \hat{P}_{*}, X_{*}, t\right)}{\partial \hat{P}_{*} \partial \hat{P}_{*}} \\
\left.\frac{\partial^{2} \Phi\left(\xi, \hat{P}_{*}, X_{*}, t\right)}{\partial \hat{P}_{*} \partial X_{*}} \frac{\partial^{2} \Phi\left(\xi, \hat{P}_{*}, X_{*}, t\right)}{\partial X_{*} \partial \hat{P}_{*}} \frac{\partial^{2} \Phi\left(\xi, \hat{P}_{*}, X_{*}, t\right)}{\partial X_{*} \partial X_{*}}\right|^{T} d \xi D,
\end{gathered}
$$

where $\hat{P}_{*}, X_{*}$ are approximated solutions of system (17);

$D$ is the matrix of the weight factors for the deviation of the approximated solution from the required one [6].

The example illustrating the feasibility of the real application of the approach given is considered in [7].

To reduce the computing expenses on the basis of the approximation of the DAPD let us consider the algorithm of the synthesis using the local criterion.

\section{The a posteriori local - optimum control for the structure selection}

Local criterion J:

$$
J=\int_{\xi_{*}} \varphi_{0}\left[\omega_{z}(\xi, t)\right] d \xi+\int_{t_{0}}^{t} \int_{\xi_{*}} v_{0}^{T}(\xi, z, t) v_{0}(\xi, z, t) d \xi d t
$$

where $\varphi_{0}$ is the non-negatively defined scalar function generally dependent already directly on vector $\omega_{z}$,

is typical for the process control in the real time.

In this case the solution is reduced to the search of such vector

$v_{0}=v_{0}(\xi, z, t)$, which would provide the minimum of criterion (18) provided that vector $\omega_{Z}(\xi, t)$ is described by the known vector equation with partial derivatives, deduced in [1] and transformed in paragraph 1 to the following form (6):

$$
\frac{\partial \omega_{z}}{\partial t}=U\left(\omega_{z}\right)-F\left(\omega_{z}\right) v_{0}
$$


where $U\left(\omega_{\mathrm{Z}}\right)$ is the vector - function representing the vector generalization of the right-hand part of the Stratonovich equation [1];

$F\left(\omega_{z}\right)$ is the matrix function linearly dependent on the component of vector $\omega_{z}$.

In the case considered this allows to obtain the expression of the minimized function $I=(-\dot{J})$ for the further definition of the optimum intensity vector for the state change of structures $v_{0}^{*}$

$$
I=-\int_{\xi_{\star}}\left(\frac{\partial \varphi_{0}}{\partial \omega_{z}} \frac{\partial \omega_{z}}{\partial t}+v_{0}^{T} v_{0}\right) d \xi
$$

or in view of the right-hand part of equation (6):

$$
I=-\int_{\xi_{*}}\left(\frac{\partial \varphi_{0}}{\partial \omega_{z}}\left[U\left(\omega_{z}\right)-F\left(\omega_{z}\right) v_{0}\right]+v_{0}^{T} v_{0}\right) d \xi
$$

From the condition of the maximum for expression (19) we have the initial equation for defining vector $v_{0}{ }^{*}$

$$
-\frac{\partial \varphi_{0}}{\partial \omega_{z}} F\left(\omega_{z}\right)+2 v_{0}^{T}=0
$$

whence the vector required

$$
v_{0}^{*}=\frac{1}{2} F^{T}\left(\omega_{z}\right) \frac{\partial \varphi_{0}^{T}}{\partial \omega_{z}} .
$$

If instead of (18) we use a less general expression, which does not impose any restrictions on the definitional domain of vector $v_{0}$, as the criterion of optimization:

$$
J_{1}=\int_{\xi_{*}} \varphi_{0}\left[\omega_{z}(\xi, t)\right] d \xi+\int_{t_{0}}^{t} v_{0}^{T}(z, t) v_{0}(z, t) d t
$$

then expression $I_{1}=\left(-\dot{J}_{1}\right)$ as compared to (19) is modified as follows:

$$
I_{1}=-\left(\int_{\xi_{*}} \frac{\partial \varphi_{0}}{\partial \omega_{z}}\left[U\left(\omega_{z}\right)-F\left(\omega_{z}\right) v_{0}\right] d \xi+v_{0}^{T} v_{0}\right)
$$

whence

$$
v_{0}^{*}=\frac{1}{2} \int_{\xi_{*}} F^{T}\left(\omega_{z}\right) \frac{\partial \varphi_{0}^{T}}{\partial \omega_{z}} d \xi
$$


The substitution of the found optimum vector $v_{0}{ }^{*}(20)$ (or (22)) in equation (6) allows to form the equation, describing the required vector $\omega_{Z}$ of the distribution densities of the extended state vectors for the system with the intensity of their change, which provides the optimum of functional (18) (or (21), respectively). So, in case of selecting as the criterion of optimality (18) we have:

$$
\frac{\partial \omega_{z}}{\partial t}=U\left(\omega_{z}\right)-\frac{1}{2} F\left(\omega_{z}\right) F^{T}\left(\omega_{z}\right) \frac{\partial \varphi_{0}^{T}}{\partial \omega_{z}},
$$

and in case of $(21)$ -

$$
\frac{\partial \omega_{z}}{\partial t}=U\left(\omega_{z}\right)-\frac{1}{2} F\left(\omega_{z}\right) \int_{\xi_{\star}} F^{T}\left(\omega_{z}\right) \frac{\partial \varphi_{0}^{T}}{\partial \omega_{z}} d \xi .
$$

It is obvious that the integration of the equations obtained completes the solution of the selection problem of the optimum structure in the sense of (18) or (21) by the subsequent definition of the maximal component of the vector of the state probabilities

$$
P(t)=\int_{-\infty}^{\infty} \omega_{z}(\xi, t) d \xi .
$$

It should be noted that from the point of view of the computing expenses the solution of equations (23), (24) appears to be not much more complicated than the initial system of the integro-differential equations (6) (a basic one in the theory of the dynamic systems of random structure [1]) and incommensurably more simple than the solution of the point-topoint boundary-value problem for two systems of the integro-differential equations with partial derivatives, given in paragraph 2. Moreover, the solution of the equations given allows to obtain in the real time the exact solution of the problem stated, while the approach, considered in paragraph 2, provides only the formation of the current suboptimum solution. For the comparative efficiency estimation of the both methods we consider the example.

As the target we choose an observable two-structural nonlinear dynamic system described in paragraph 2, for which functions $U\left(\omega_{\mathrm{z}}\right), F\left(\omega_{\mathrm{z}}\right)$ determining the right-hand part of equation (6) have the form:

$$
U\left(\omega_{z}\right)=\left|\begin{array}{c}
\frac{\partial}{\partial \xi}\left(\xi^{2} \omega_{z}^{(1)}\right)+\frac{1}{2} \frac{\partial^{2} \omega_{z}^{(1)}}{\partial \xi^{2}}-\frac{1}{2} \omega_{z}^{(1)}\left[\left(z-0,5 \xi^{2}\right)^{2}-\sum_{k=1}^{2} \int_{-\infty}^{\infty}\left(z-0,5 x^{2}\right)^{2} \omega_{z}^{(k)}(x, t) d x\right. \\
\frac{\partial}{\partial \xi}\left[\left(\xi-0,01 \xi^{3}\right) \omega_{z}^{(2)}\right]+\frac{1}{2} \frac{\partial^{2} \omega_{z}^{(2)}}{\partial \xi^{2}}-\frac{1}{2} \omega_{z}^{(2)}\left[\left(z-1,2 \xi^{2}+0,1 \xi^{3}\right)^{2}-\right. \\
\left.-\sum_{k=1}^{2} \int_{-\infty}^{\infty}\left(z-1,2 x^{2}+0,1 x^{3}\right)^{2} \omega_{z}^{(k)}(x, t) d x\right]
\end{array}\right|
$$




$$
F\left(\omega_{z}\right)=\left|\begin{array}{cc}
\omega_{z}^{(1)} & -\omega_{z}^{(2)} \\
-\omega_{z}^{(1)} & \omega_{z}^{(2)}
\end{array}\right|
$$

where $z$ is the output signal of the nonlinear observer of process $\xi$.

As far as according to the constrains of the example given in paragraph 2, the selection of the process structure is required to be carried out, proceeding from the provision of the probability maximum of its occurrence in the given limits $\xi_{*}=\left[\xi_{\min }=-0,5 ; \xi_{\max }=0,7\right]$, then for forming the non-negatively defined criterion function $\varphi_{0}$ in (18), ensuring in minimizing $J$ the performance of the given criterion, we accomplish the following additional constructions.

The condition of providing the maximum of probability $P(t)=\int_{\xi_{\star}} \omega_{z}(\xi, t) d \xi$ is equivalent to that of the minimum $\left(I_{S}^{T}-P\right)$ or $\left(I_{S}-P^{T}\right)\left(I_{S}^{T}-P\right)$, where $I_{S}$ is the unit row of dimension $S$. Evaluating the last expression, we have

$$
\begin{aligned}
& \left(I_{S}-P^{T}\right)\left(I_{S}^{T}-P\right)=I_{S} I_{S}^{T}-P^{T} I_{S}^{T}-I_{S} P+P^{T} P=S-2 I_{S} P+P^{T} P= \\
& =S \int_{\xi_{\star}} \frac{d \xi}{\xi_{\max }-\xi_{\min }}-2 I_{S} \int_{\xi_{\star}} \omega_{z}(\xi, t) d \xi+\int_{\xi_{\star}} \omega_{z}^{T}(\xi, t) d \xi \int_{\xi_{\star}} \omega_{z}(\xi, t) d \xi .
\end{aligned}
$$

As far as by virtue of the Cauchy-Bunyakovsky inequality

$$
\int_{\xi_{*}} \omega_{z}^{T}(\xi, t) d \xi \int_{\xi_{*}} \omega_{z}(\xi, t) d \xi \leq \int_{\xi_{*}} \omega_{z}^{T}(\xi, t) \omega_{z}(\xi, t) d \xi\left(\xi_{\max }-\xi_{\min }\right),
$$

then after replacing the last summand by the right-hand part of the given inequality in expression (25), the function (25) remains non-negatively defined. Presenting expression (25) transformed in the form

$$
\int_{\xi_{*}}\left(\frac{S}{\xi_{\max }-\xi_{\min }}-2 I_{S} \omega_{z}(\xi, t)+\left(\xi_{\max }-\xi_{\min }\right) \omega_{z}^{T}(\xi, t) \omega_{z}(\xi, t)\right) d \xi,
$$

we obtain the required criterion function $\varphi_{0}$ as follows:

$$
\varphi_{0}\left(\omega_{z}\right)=S A^{-1}-2 I_{S} \omega_{z}+A \omega_{z}^{T} \omega_{z}
$$

where $A=\xi_{\max }-\xi_{\min }$.

In this case equation (23) for the optimum vector $\omega_{Z}$ takes the form 


$$
\begin{gathered}
\frac{\partial \omega_{z}}{\partial t}=U\left(\omega_{z}\right)-F\left(\omega_{z}\right) F^{T}\left(\omega_{z}\right)\left(\begin{array}{c}
\mid \omega_{z}^{(1)} \\
\omega_{z}^{(2)}
\end{array} \mid A-I_{S}^{T}\right)= \\
=U\left(\omega_{z}\right)-\left|\begin{array}{c}
\omega_{z}^{(1) 3}+\omega_{z}^{(1)} \omega_{z}^{(2)}\left(\omega_{z}^{(2)}-\omega_{z}^{(1)}\right)-\omega_{z}^{(2) 3} \\
-\omega_{z}^{(1) 3}+\omega_{z}^{(1)} \omega_{z}^{(2)}\left(\omega_{z}^{(1)}-\omega_{z}^{(2)}\right)+\omega_{z}^{(2) 3}
\end{array}\right| \cdot 1,2 .
\end{gathered}
$$

The solution of the given equation was carried out similarly to paragraph 2 on the basis of

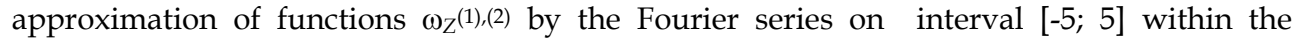
accuracy of 4 terms of expansion and integration of the obtained system of equations for the expansion factors on the time interval $[0 ; 300] \mathrm{s}$. When integration and formation of the approximated values of functions $\omega Z^{(1)}, \omega Z^{(2)}$ had been taken place, the numbers of structures, chosen by the character of the maximal state probability at the current time, appeared to be distributed in time as follows:

- in interval $[0 ; 53] \mathrm{s}$ - the second structure;

- in interval $[53 ; 119] \mathrm{s}$-the first structure;

- in interval $[119 ; 300] \mathrm{s}$-the second structure.

The comparative analysis of the solution obtained with that of suggested in paragraph 2 shows that with their practically identical accuracy (upon completing the simulation the difference of the probability values for occurring process $\xi$ in the limits $\xi^{*}$ was less than $7 \%$ ) the computing expenses were reduced rather sufficiently in the case considered: the volume of the operative memory, required for the solution, has been decreased by a factor of $\sim 3,5$, the solution time - by a factor of $\sim 2,1$.

Thus, despite of the less generality from the point of view of the theory, the local control in the real multistructural systems has obvious advantages over the terminal one (especially in the systems using the real-time measuring information). In summary we consider the investigated problem of the control synthesis by the local criterion on the basis of applying the approximating representations of the distribution density.

\section{Suboptimum structure control on the basis of the local generalized criterions}

In this case taking into account the character of the criterions given in paragraphs $3 ; 4$ and preliminary reasoning the minimized local criterion $J$ is written down in the following form:

$$
J=\int_{\xi_{\star}} \varphi_{0}[\tilde{\rho}(\xi, \hat{p}, x, t)] d \xi+\int_{t_{0}}^{t} v_{0}^{T}(\hat{p}, x, t) v_{0}(\hat{p}, x, t) d t
$$

where $\varphi_{0}$ is the non-negatively defined scalar function. 
In this case the solution is reduced to the search of such vector $v_{0}=v_{0}(\hat{p}, x, t)$, which would provide the minimum of criterion (26) given that the extended vector $\left|\begin{array}{l}\hat{p} \\ x\end{array}\right|$ is described by the known vector equation derived in [1] and transformed in paragraph 3 to the following form (16):

$$
\begin{gathered}
\left|\begin{array}{c}
\hat{\dot{p}} \\
\dot{x}
\end{array}\right|=A(x, t)+B(\hat{p}, x) v_{0}, \\
A(x, t)=\left|\begin{array}{c}
0 \\
\varphi(x, t)
\end{array}\right|, B(\hat{p}, x)=\left|\begin{array}{c}
\left(E \otimes \hat{p}^{T}\right)-\hat{p}\left(E \otimes I_{S}\right) E_{1} \\
T(\hat{p}, x)
\end{array}\right| E_{0} .
\end{gathered}
$$

The final solution of the problem stated - the selection of the number of the structure, providing the optimum of functional (26) at the real time, is carried out similarly to that of given in paragraph 3 by solving equation (16) in case of the found optimum law $v_{0}^{*}$ - for construct vector $\hat{p}$ and define its maximal component, the number of which will define the number of the structure required for the state vector. Following the stated above in paragraphs 3,4 , in this case the optimum function $v_{0}^{*}$ is synthesized from the constrain

$$
\max _{v_{0}}\left[-\left(\frac{\partial}{\partial t} \int_{\xi_{\star}} \varphi_{0}[\tilde{\rho}(\xi, \hat{p}, x, t)] d \xi+v_{0}^{T}(\hat{p}, x, t) v_{0}(\hat{p}, x, t)\right],\right.
$$

which, in its turn, results in the initial equation with respect to $v_{0}^{*}$ :

$$
\frac{\partial}{\partial v_{0}} \int_{\xi_{\star}}\left(\frac{\partial}{\partial t} \varphi_{0}[\tilde{\rho}]\right) d \xi+2 v_{0}^{* T}=0
$$

Due to the fact that

$$
\frac{\partial}{\partial t} \varphi_{0}[\tilde{\rho}]=\frac{\partial \varphi_{0}}{\partial \tilde{\rho}}\left\{\left|\frac{\partial \tilde{\rho}}{\partial \hat{p}}: \frac{\partial \tilde{\rho}}{\partial x} \| \begin{array}{l}
\hat{\dot{p}} \\
\dot{x}
\end{array}\right|\right\}
$$

and vector $\left|\begin{array}{l}\hat{p} \\ x\end{array}\right|$ is described by the system of equations (16), then we obtain the following equation with respect to $v_{0}^{*}$ : 


$$
\frac{\partial}{\partial v_{0}}\left\{\int_{\xi_{*}} \frac{\partial \varphi_{0}}{\partial \tilde{\rho}}\left|\frac{\partial \tilde{\rho}}{\partial \hat{p}}: \frac{\partial \tilde{\rho}}{\partial x}\right| d \xi\left(A+B v_{0}^{*}\right)\right\}+2 v_{0}^{* T}=0
$$

Finally from the expression given we have

$$
\int_{\xi_{*}} \frac{\partial \varphi_{0}}{\partial \tilde{\rho}}\left|\frac{\partial \tilde{\rho}}{\partial \hat{p}}: \frac{\partial \tilde{\rho}}{\partial x}\right| d \xi B+2 v_{0}^{*}=0
$$

whence the optimum law $v_{0}^{*}$ is immediately defined:

$$
v_{0}^{*}=-\frac{1}{2} B^{T} \int_{\xi_{\star}}\left|\frac{\partial \tilde{\rho}}{\partial \hat{p}}: \frac{\partial \tilde{\rho}}{\partial x}\right| \frac{\partial \varphi_{0}}{\partial \tilde{\rho}} d \xi
$$

The substitution (27) into (16) results in the required equations describing the evolution of the parameters of the distribution densities for the state vectors in the structures and the probabilities of occurring of those for the system optimum in the sense of (26):

$$
\left|\begin{array}{c}
\hat{\dot{p}} \\
\dot{x}
\end{array}\right|=A(\hat{p}, x, t)-\frac{1}{2} B(\hat{p}, x) B^{T}(\hat{p}, x) \int_{\xi_{\star}}\left|\frac{\partial \tilde{\rho}}{\partial \hat{p}}: \frac{\partial \tilde{\rho}}{\partial x}\right| \frac{\partial \varphi_{0}}{\partial \tilde{\rho}} d \xi .
$$

Then the structure selection is carried out similarly to that of stated above on the basis of defining the number of the maximal component for vector $\hat{p}$ being sufficiently trivial operation, which does not practically affect the total volume of the computing expenses. Despite some reduction in the generality of the solution considered in comparison, for example, with the similar terminal one, obtained in paragraph 3, its obvious advantage is the absence of the need for the solution of the point-to-point boundary-value problem and, as a consequence, the feasibility to apply the optimum solution simultaneously with the essential reduction of the computing expenses for the real systems as compared to the general case.

So, the comparative analysis of the solution obtained with that of suggested in paragraph 3 , which has been carried out on the basis of the numerical simulation, has shown that with their practically identical accuracy (upon completing the simulation the difference of values for the probabilities of occurring process $\xi$ within the limits of interval $\xi_{*}$ was less than $9 \%$ ) the computing expenses in the considered case were reduced rather essentially: the volume of the operative memory required for their solution, has decreased by a factor of $\sim 3,7$, the solution time -by a factor of 2,4 .

The practical recommendations here are obvious and do not require the additional comments. 


\section{References}

1. Kazakov I.E., Artemiev V.M. Optimization of dynamic systems with random structure.Moscow, "Science", 1980.

2. Xu X., Antsaklis P.J. Optimal control of switched systems based on parameterization of the switching instants. // IEEE Trans. Automat. Contr., v.49, no.1, pp.2-16, 2004.

3. Sirazetdinov T.K. Optimization of systems with distributional parameters. - Moscow, "Science", 1977.

4. Pugachev V.S., Sinitsyn I.N. Stochastic differential systems. - Moscow, "Science", 2004.

5. Krasovsky A.A. and other. Theory of the automatic control (reference book). - Moscow, "Science", 1987.

6. Pervachev S.V., Perov A.I. Adaptive filtration of messages. - Moscow, "Radio and communication", 1991.

7. S.V. Sokolov, Yu.I. Kolyada, F.V. Mel'nichenko. Optimal control of nonlinear stochastic structures. // Automatic Control and Computer Sciences. Allerton Press Inc., New York, v.32, no.2, pp.18-25, 1998. 


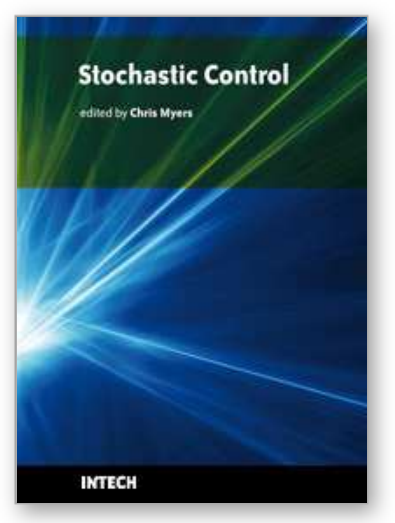

\author{
Stochastic Control \\ Edited by Chris Myers
}

ISBN 978-953-307-121-3

Hard cover, 650 pages

Publisher Sciyo

Published online 17, August, 2010

Published in print edition August, 2010

Uncertainty presents significant challenges in the reasoning about and controlling of complex dynamical systems. To address this challenge, numerous researchers are developing improved methods for stochastic analysis. This book presents a diverse collection of some of the latest research in this important area. In particular, this book gives an overview of some of the theoretical methods and tools for stochastic analysis, and it presents the applications of these methods to problems in systems theory, science, and economics.

\title{
How to reference
}

In order to correctly reference this scholarly work, feel free to copy and paste the following:

Sergey V. Sokolov (2010). The Synthesis Problem of the Optimum Control for Nonlinear Stochastic Structures in the Multistructural Systems and Methods of Its Solution, Stochastic Control, Chris Myers (Ed.), ISBN: 978953-307-121-3, InTech, Available from: http://www.intechopen.com/books/stochastic-control/the-synthesisproblem-of-the-optimum-control-for-nonlinear-stochastic-structures-in-the-multistructu

\section{INTECH}

open science | open minds

\section{InTech Europe}

University Campus STeP Ri

Slavka Krautzeka 83/A

51000 Rijeka, Croatia

Phone: +385 (51) 770447

Fax: +385 (51) 686166

www.intechopen.com

\section{InTech China}

Unit 405, Office Block, Hotel Equatorial Shanghai

No.65, Yan An Road (West), Shanghai, 200040, China

中国上海市延安西路65号上海国际贵都大饭店办公楼 405 单元

Phone: +86-21-62489820

Fax: $+86-21-62489821$ 
(C) 2010 The Author(s). Licensee IntechOpen. This chapter is distributed under the terms of the Creative Commons Attribution-NonCommercialShareAlike-3.0 License, which permits use, distribution and reproduction for non-commercial purposes, provided the original is properly cited and derivative works building on this content are distributed under the same license. 